\title{
UV Radiation in Different Stellar Systems
}

\author{
A. P. Buccino \& P. J. D. Mauas \\ IAFE (CONICET-UBA), CC. 67, Suc. 28, 1428 Buenos Aires, \\ Argentina \\ G.A. Lemarchand \\ IAR (CONICET) and CEA (UBA). C.C. 8, Suc. 25, 1425 Buenos \\ Aires, Argentina
}

\begin{abstract}
We have studied the UV radiation in the range 180 to $330 \mathrm{~nm}$ emitted by several stars with planetary systems, in order to determine the constraints on the habitable zones posed by the radiation levels. The observations were taken from the International Ultraviolet Explorer (IUE) and the Hubble Space Telescope (HST) satellites database. The stellar sample was chosen to cover the range of what are usually called solartype stars, from late $\mathrm{F}$ to $\mathrm{K}$ stars. We found that the planets discovered around Epsilon Eridani and 16 Cyg B are within both the optical and UV habitable zones.
\end{abstract}

\section{Introduction}

When we speculate about the possibility of life elsewhere in the universe, we are assuming that the hypothetical extraterrestrial biospheres have environmental and evolutionary conditions similar to those seen on Earth. In other words, we assume that the most likely places to find evidence of life beyond Earth are those that have similar physical parameters to our home planet.

Therefore, the main target for these kind of studies are the solar-type stars, which are similar to the Sun in mass and evolutionary state. In practical terms, Soderblom \& King (1998) defined "solar-like" as main sequence stars of spectral class F8V to K2V (or B-V within 0.50 to 1.00 ). Several extrasolar planets have been found around those types of stars, so an important point to study is whether they are suitable for life or not.

Stellar luminosity and its evolution in time are dominant factors in determining the location and the size of circumstellar habitable zones. There are many definitions of habitable zones (HZ); in this work we use Kasting's definition of $\mathrm{HZ}$ as the region in which a planet can maintain liquid water on its surface (Kasting 1996). Using one-dimensional radiative-convective climate models, Kasting et al. (1993) estimated the boundaries of the HZ. The inner one was defined by the "loss of water" from the planet by either the moist or runaway greenhouse effect and the outer one by the freezing of all water in the planet's surface. Habitable zones tend to migrate outward with time because main sequence stars become brighter as they age and convert hydrogen into helium and heavier elements. 
In this work we introduce a second boundary condition to analyse the $\mathrm{HZ}$ extension around extrasolar planets: the flux of the UV radiation. This second criterion is based in the fact that the life on a planet may be at risk because of the biologically damaging effects of the UV radiation, which is known to inhibit photosynthesis, induce DNA damage, and cause damage in a wide variety of proteins and lipids. UV radiation below $290 \mathrm{~nm}$ becomes energetically very damaging to most biological systems (Lindberg \& Horneck 1991).

Optical and UV habitable zones within extrasolar planetary systems

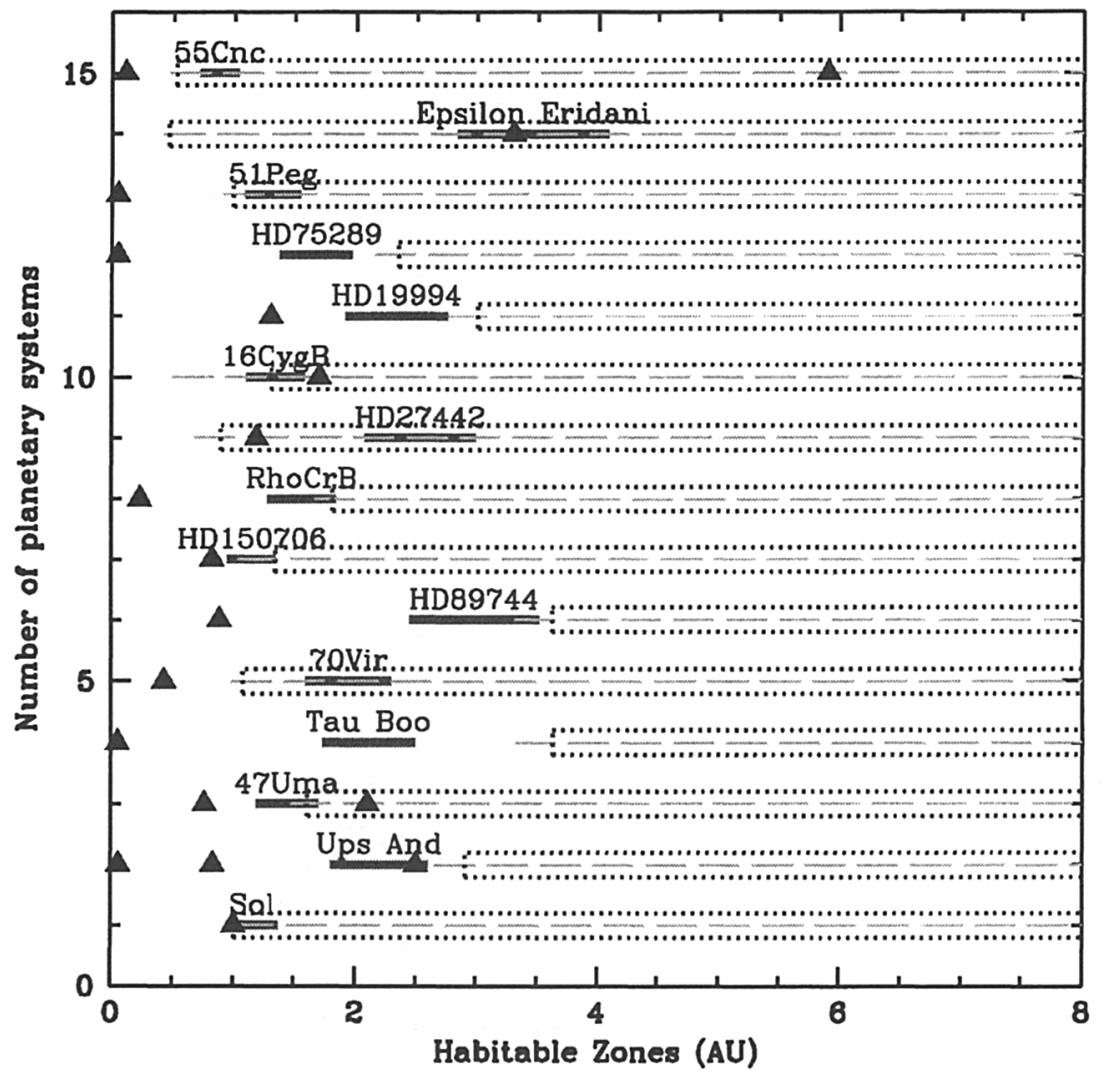

Figure 1. Habitable zones for visible and UV radiation

\section{UV Boundaries for Habitable Zones}

Kasting et al. (1997) argue that early-type stars will produce large amounts of UV that may be harmful for life, but they also recognize that late-type stars, which emit proportionally less radiation at short wavelengths $(\lambda<200 \mathrm{~nm})$ will be less efficient to split $\mathrm{O}_{2}$ and initiate ozone formation. In their analysis they also show that Earth-like planets orbiting $\mathrm{F}$ and $\mathrm{K}$ stars may well receive less harmful radiation at their surfaces than does the Earth itself.

UV radiation is usually divided in three wavelength ranges; UVA (315$400 \mathrm{~nm}), \operatorname{UVB}(280-315 \mathrm{~nm}$ ) and UVC (below $280 \mathrm{~nm}$ ). It is widely accepted that in the archean Earth (3.9-2.5 Gyr), prior to the building up of atmospheric 
oxygen and ozone, UVC and high levels of UVB would probably have reached the surface of the Earth relatively unattenuated (Sagan 1973; Kasting 1993).

From the biologically effective irradiance data, it could be expected that about $65 \%$ of the biological response to DNA on archean Earth is accounted for by UVC. On the other hand, Cockell (1998) showed that protection from the harmful effects of UV radiation is a requirement for many organisms, particularly photosynthetic organisms that depend upon exposure to sunlight for their way of life. In particular, the non-specific absorption of UVC below $240 \mathrm{~nm}$ by many macromolecules may generate a mechanism to protect DNA inside the cell from a significant part of the UVC. Garcia-Pichel (1998) has recently concluded that UVB will exert a more important effect on early Earth.

By the end of June 2002, 86 planetary systems have been discovered, with 99 extrasolar planets and 11 multiple planetary systems (Schneider 2002). From this sample we consider 15 planetary systems listed in Table 1 . We analyzed spectra obtained with the International Ultraviolet Explorer and the Hubble Space Telescope Satellites to obtain the levels of UV radiation between 185 to $320 \mathrm{~nm}$. In Table 1 we list the different values of the UVC, UVB and UVA that reach each discovered planet and we compare these values with those at the Early and Present Earth.

Table 1: UV radiation around a sample of 18 exoplanets

\begin{tabular}{|c|c|c|c|c|}
\hline $\operatorname{Star} N^{\circ}$ & Planets around Stars & $\begin{array}{r}\text { UVC } \\
(200-280 n m) \\
\left(\mathrm{W} / \mathrm{m}^{2}\right) \\
\end{array}$ & $\begin{array}{r}\text { UVB } \\
(280-315 n m) \\
\left(\mathrm{W} / m^{2}\right) \\
\end{array}$ & $\begin{array}{r}\text { UVA } \\
(315-400 n m) \\
\left(\mathrm{W} / m^{2}\right) \\
\end{array}$ \\
\hline 1 & Early Earth $(3.8 \mathrm{Gyr})$ & 3.10 & 11.70 & 56.30 \\
\hline 1 & Present Earth & 0.00 & 3.70 & 71.30 \\
\hline 2 & Ups And planet A & 7537.60 & 22608.36 & 7759.29 \\
\hline 2 & Ups And planet B & 38.09 & 114.24 & 39.21 \\
\hline 2 & Ups And planet $\mathrm{C}$ & 4.20 & 12.59 & 4.32 \\
\hline 3 & 47Uma planet $\mathrm{A}$ & 1.82 & 6.38 & 2.61 \\
\hline 3 & 47Uma planet $\mathrm{B}$ & 13.89 & 48.72 & 19.94 \\
\hline 4 & Tau Boo planet A & 19131.46 & 39987.91 & 12971.00 \\
\hline 5 & 70Vir planet $\mathrm{A}$ & 19.18 & 107.63 & 43.90 \\
\hline 6 & HD89744 planet A & 52.51 & 181.77 & 94.63 \\
\hline 7 & HD150706 planet $A$ & 5.85 & 31.36 & 29.44 \\
\hline 8 & RhoCrB planet $\mathrm{A}$ & 192.00 & 610.68 & 249.16 \\
\hline 9 & HD27742 planet A & 1.18 & 6.69 & 4.52 \\
\hline 10 & 16CygB planet A & 1.81 & 6.40 & 2.66 \\
\hline 11 & HD19994 planet A & 16.61 & 47.32 & 17.71 \\
\hline 12 & HD75289 planet A & 8058.06 & 19631.41 & 7596.54 \\
\hline 13 & 51Peg planet $\mathrm{A}$ & 1207.69 & 5019.48 & 2216.60 \\
\hline 14 & Eps Eri planet A & 0.06 & 0.14 & 0.0 \\
\hline 15 & $55 \mathrm{Cnc}$ planet $\mathrm{A}$ & 64.71 & 256.59 & 141.3 \\
\hline 15 & $55 \mathrm{Cnc}$ planet $\mathrm{B}$ & 0.02 & 0.09 & 0.0 \\
\hline
\end{tabular}

Assuming the so-called "Principle of Mediocrity" (von Hoerner 1961) we set the $\mathrm{HZ}$ for $\mathrm{UV}$ radiation in the following way. We consider the UV radiation incident on the Earth of 3.8 Gyr ago, estimated by Cockell (1998), to set an upper limit to the accepted levels of radiation. In this way we obtain a pessimistic minimum boundary for the HZ, which is shown by the dotted frame in Fig. 1. 
From the present levels of UV radiation at the Earth's orbit, which allow the presence of complex life with the existence of a planetary atmosphere rich in ozone, we have also calculated the UV habitable zones for complex life. We show this HZ for Earth-like planets with a dashed line. Also shown in Fig. 1, as a solid line, is the HZ found with the traditional definition based on the levels of visible radiation (Kasting 1996). For each star, we obtained the visible flux from the visual magnitude $\left(m_{V}\right)$, and computed the region where the stellar flux would be similar to the solar one at 0.95 and $1.37 \mathrm{AU}$. Finally, the triangles in Fig. 1 mark the positions of the discovered planets.

\section{Discussion}

In a recent study, Noble et al. (2002) found that long-term orbital stability of Earth-like planets in stellar HZ is possible for the cases of $70 \mathrm{Vir}$ and 51 Peg, even though they cannot be detected with the present telescopes. On the other hand, from theoretical dynamical considerations, they also found that the presence of Earth-like planets within the UV and optical HZ of Gl 876, Upsilon And and HD210277 can be ruled out.

The figure shows that the planet which orbits Epsilon Eridani fits both criteria, optical and UV, for the HZ, indicating that it may have liquid water on its surface, and that the levels of UV radiation are similar to those on Earth. However, this is not a terrestrial-type planet $\left(M \sin (i)=0.86 M_{J}\right)$. We also point out that the existence of this particular planet remains controversial, since this is a particularly active star, and the Doppler behavior of magnetically active stars remains poorly explored (Marcy et al. 2000).

Another interesting candidate is the planet discovered around 16 Cyg B. Even though this Jupiter-like planet $\left(M \sin (i)=1.5 M_{J}\right)$ is out of the optical $\mathrm{HZ}$ it is close enough to allow the existence of liquid water generated by some other atmospheric mechanisms.

\section{References}

Cockell, C. S. 1998, J. theor. Biol., 193, 717

García-Pichel, F. 1998, Orig. Life Evol. Biosph., 23, 321

Kasting, J. F. 1993, Science, 259, 920

Kasting, J. F. 1996, in Circumstellar Habitable Zones, ed. L. R. Doyle, (Menlo Park: Travis)

Kasting, J. F., Whitmire D. P., \& R. T. Reynolds 1993, Icarus, 101, 108

Kasting, J. F., Whittet D. C. D., \& Sheldon, W. R. 1997, Orig. Life Evol. Biosph., 27, 413

Lindberg, C., \& Horneck G. 1991, J. Photochem. Photobiol. B: Biol., 11, 69

Marcy, G. W., Cochran, W. D., \& Mayor, M. 2000, in Protostars and Planets, ed. V. Mannings, et al., (Tucson: Univ. of Arizona Press), 1285

Noble, M., Musielak, Z. E., \& Cuntz, M. 2002, ApJ 572, 1024

Sagan, C. 1973, J. theor.Biol., 39, 195

Schneider, J. 2002, Extra-solar Planets Catalog, cfa-www.harvard.edu/planets Soderblom, D., \& King, A. 1998, in Solar Analogs, Proc. of $2^{\text {nd }}$ Lowell Workshop von Hoerner, S. 1961, Science, 134, 1839 